\title{
A Cost-Utility Analysis Comparing Oncoplastic Breast Surgery to Standard Lumpectomy in Large Breasted Women
}

\author{
Abhishek Chatterjee1, Anaeze C. Offodile II', Ammar Asban'3, Raquel A. Minasian', \\ Albert Losken", Roger Graham ${ }^{1}$, Lilian Chen ${ }^{1}$, Brian J. Czerniecki ${ }^{5}$, Carla Fisher6 \\ ${ }^{1}$ Department of Surgery, Tufts University Medical Center, Boston, MA, USA \\ ${ }^{2}$ Johns Hopkins Bloomberg School of Public Health, Baltimore, MD, USA \\ ${ }^{3}$ Department of Surgery, University of Alabama at Birmingham, Birmingham, AL, USA \\ ${ }^{4}$ Division of Plastic and Reconstructive Surgery, Department of Surgery, Emory University School of Medicine, Atlanta, GA, USA \\ ${ }^{5}$ Department of Breast Oncology, H Lee Moffitt Cancer Center, Tampa, FL, USA \\ ${ }^{6}$ Department of Surgery, University of Pennsylvania, Philadelphia, PA, USA \\ Email:ammarasban@gmail.com
}

How to cite this paper: Chatterjee, A., Offodile II, A.C., Asban, A., Minasian, R.A., Losken, A., Graham, R., Chen, L., Czerniecki, B.J. and Fisher, C. (2018) A Cost-Utility Analysis Comparing Oncoplastic Breast Surgery to Standard Lumpectomy in Large Breasted Women. Advances in Breast Cancer Research, 7, 187-200.

https://doi.org/10.4236/abcr.2018.72011

Received: April 1, 2018

Accepted: April 27, 2018

Published: April 30, 2018

Copyright (c) 2018 by authors and Scientific Research Publishing Inc. This work is licensed under the Creative Commons Attribution International License (CC BY 4.0).

http://creativecommons.org/licenses/by/4.0/ (c) (i) Open Access

\begin{abstract}
Purpose: Ablative options, beyond mastectomy, for large breasted patients with breast cancer include oncoplastic resection via reduction pattern and standard lumpectomy. Oncoplastic resection also entails a contralateral procedure for symmetry and the potential benefit of a superior cosmetic outcome. Our aim was to examine the cost-effectiveness of this treatment strategy comparing it to standard lumpectomy in treating breast cancer patients. Methods: A literature review was performed of the probabilities and outcomes related to treatment of unilateral breast cancer via oncoplastic resection or unilateral lumpectomy. Utility score surveys were used to estimate the quality adjusted life years (QALYs) associated with a successful procedure, additional margins excision and post-operative complications. A decision analysis tree was developed to highlight the more cost-effective strategy. An Incremental Cost-Utility Ratio (ICUR) was calculated. Sensitivity analysis was performed to check the robustness of our data. Results: Oncoplastic resection was associated with fewer positive margins relative to standard lumpectomy $(10.0 \%$ versus $18 \%$ ). In cases with positive margins, a greater percentage of oncoplastic resection patients chose a mastectomy compared to the lumpectomy patients (72\% versus $19 \%)$. Utility scores for a successful operation favored oncoplastic resection (92.6 versus 86.55), but in instances of positive margins, favored the lumpectomy patients (74.2 versus 70.2). Decision tree analysis revealed that oncoplastic resection was more cost-effective with an ICUR of \$2609.66/QALY gained. Conclusion: Oncoplastic resection represents a
\end{abstract}


cost-effective strategy for the large breasted patient and provides the surgical team yet another reasonable option for the appropriate patient.

\section{Keywords}

Breast Cancer, Lumpectomy, Mastectomy, Cost-Utility Analysis

\section{Introduction}

In the realm of breast conservation therapy in large breasted patients, two treatment options include standard lumpectomy (SL) versus large volume displacement oncoplastic surgery (LVOS). Each treatment varies with regards to clinical outcomes, such as positive margin rates [1] [2] and postoperative complication rates [3] [4] [5]. Variability in operative technique such as the need to operate on one versus both breasts leads to variation in costs. For this reason, we aimed to perform a cost-utility analysis to see if there is cost-utility benefit in performing SL versus LVOS when treating unilateral breast cancer in large breasted patients. Both SL and LVOS have variable clinical outcomes and costs. A meta-analysis comparing clinical outcomes between SL and LVOS was published by one of the co-authors (Losken et al., 2014) [2]. There has been no cost-utility or effectiveness analysis performed in the literature comparing SL to LVOS. This invites the opportunity to compare these two treatment options using the study of cost-utility. Cost-utility analysis considers costs, probabilities, and utilities of various health outcomes (health states) as a means to evaluate competing interventions [6]-[14].

\section{Patients and Methods}

\subsection{Perspective}

A third party payer perspective was adopted for decision analysis. This is a well-established perspective used in multiple empirical cost-utility studies [8] [9] [10] [11] that provides a fair and equally balanced cost burden to each treatment option studied.

\subsection{Literature Review and Health States}

A systematic review published by one of the authors (Losken et al. 2014) accumulated complication rates and clinical outcomes between LVOS and SL [2]. Twenty-four LVOS papers and 20 SL papers met inclusion and exclusion criteria and were analyzed. The clinically relevant positive margin rate (patients requiring additional margin resection or completion mastectomy) was 10.9\% (163/1522 patients) for LVOS and 18.0\% (520/2882 patients) for SL. Of the 163 LVOS patients requiring further surgery, 45 (27.6\%) had additional re-resection and $118(72.4 \%)$ had completion mastectomy. Of the 520 SL patients requiring further surgery, 421 (81.0\%) underwent re-excision and 99 (19.0\%) had comple- 
tion mastectomy. Early postoperative complication rates were obtained using a literature review. Large volume displacement oncoplastic surgery involves a greater amount of dissection and hence a higher complication rate (17\%) [4] [5] compared to SL (2\%) [3]. Complications included seroma, hematoma, skin necrosis and revision surgery, which presumed the need for debridement and associated costs. Positive margin rates for each approach in addition to completion mastectomy rates were included in our decision tree analysis. These clinical outcomes were defined as "health states" with associated probabilities, costs, and utilities for use in the decision model.

The template is used to format your paper and style the text. All margins, column widths, line spaces, and text fonts are prescribed; please do not alter them. You may note peculiarities. For example, the head margin in this template measures proportionately more than is customary. This measurement and others are deliberate, using specifications that anticipate your paper as one part of the entire journals, and not as an independent document. Please do not revise any of the current designations.

\subsection{Cost}

Medicare current procedure terminology (CPT) and diagnosis related groups (DRG) codes were used to assess the costs for successful oncologic and reconstructive breast surgeries, and complications including positive margins requiring further surgery including completion mastectomy. Mastectomy endpoints presumed implant reconstruction with associated costs. Costs for a complication included the cost of a successful surgery plus the cost of the particular complication. Costs for the contralateral "balancing" operation i.e. mastopexy were included as this is performed contemporaneously with LVOS. All payment data was based on 2014 Medicare CPT and DRG reimbursement national averages. [15] [16].

\subsection{Recovery}

Assumptions were made regarding appropriate follow-up for health states based on current practice of surgeons at our institutions. In terms of complications, patients who had a positive tumor margin requiring additional surgery were presumed to recover within 4 weeks of experiencing this complication. While recovery could vary based on complications, the time length associated to each complication was the same for each treatment arm in the decision tree.

\subsection{Probabilities}

The probabilities for each health state associated with clinical outcomes were taken from a comprehensive literature review published by one of the authors [2]. This data was cross-referenced with other literature reviews to establish consistency of health state outcomes and associated probabilities [1] [17].

\subsection{Utilities}

Twenty surgical experts familiar with performing breast surgery and treating 
associated postoperative complications were surveyed. These surgical experts were all practitioners of oncoplastic surgery and breast surgery in the United States, and managed complications associated to these operations. To increase generalizability, surveys were multi-institutional (9 total) with each institution having a comprehensive breast cancer center. Half of the surveys were performed by fellowship trained breast surgical oncologists and half by plastic surgeons. Utilities were obtained using visual scales. These experts were posed with identical scenarios involving each health state (but different surgical approaches) and were asked to rank their preferences such that the quality of life for each health state was marked on a "feeling thermometer", a vertical ladder of 100 units ascending from a score of 0 (death) to 100 (perfect health). The overall utility of each health state was obtained by averaging the expert opinion. Health state utility score values amongst institutions and breast surgical specialties had minimal variation with a standard deviation score range of 6 to 13 amongst questions asked.

Utilities were then converted to quality-adjusted life years (QALY) by multiplying the utility of a specific health state with the health state duration ( 4 weeks) and adding the sum of the two to the remaining life years multiplied by the utility of a successful procedure. The value of remaining life years is based on the assumptions, previously published, that a female operative candidate for breast reconstruction has a life expectancy of 81.1 years (per National Center for Health Statistics) and is 45 years old with a total life expectancy of 36.1 years. The positive margin health state, for example, assumes the positive margin "complication" would be identified, operatively corrected and recovered in a 4 week period. The average utility for experiencing a "positive margin" is 0.742 , and the utility of a successful surgery without complication is 0.8655 .

Number of health years remaining = average life expectancy - average age of patient

$$
81.8 \text { years }-45 \text { years }=36.1 \text { years }
$$

Duration of health state:

$$
4 \text { weeks } / 52 \text { weeks }=0.08 \text { years }
$$

QALY:

(utility of health state $) \times($ duration of health state $)+$ (utility of successful procedure $) \times$ (remaining life years)

$$
(0.742)(0.08)+(0.8655)(36.1-0.08)=31.23 \text { \$/QALYs }
$$

\subsection{Decision Analysis}

A decision model was created for this data (Figure 1) with the two main branches (or arms of the decision tree) being oncoplastic surgery and breast conservation surgery (standard lumpectomy) for treatment of breast cancer in ptotic or large breasted women. The costs and QALYs for each health state were incorporated along with the probabilities of all health states relevant to the surgery. Expected values for costs and outcomes were derived by multiplying the 


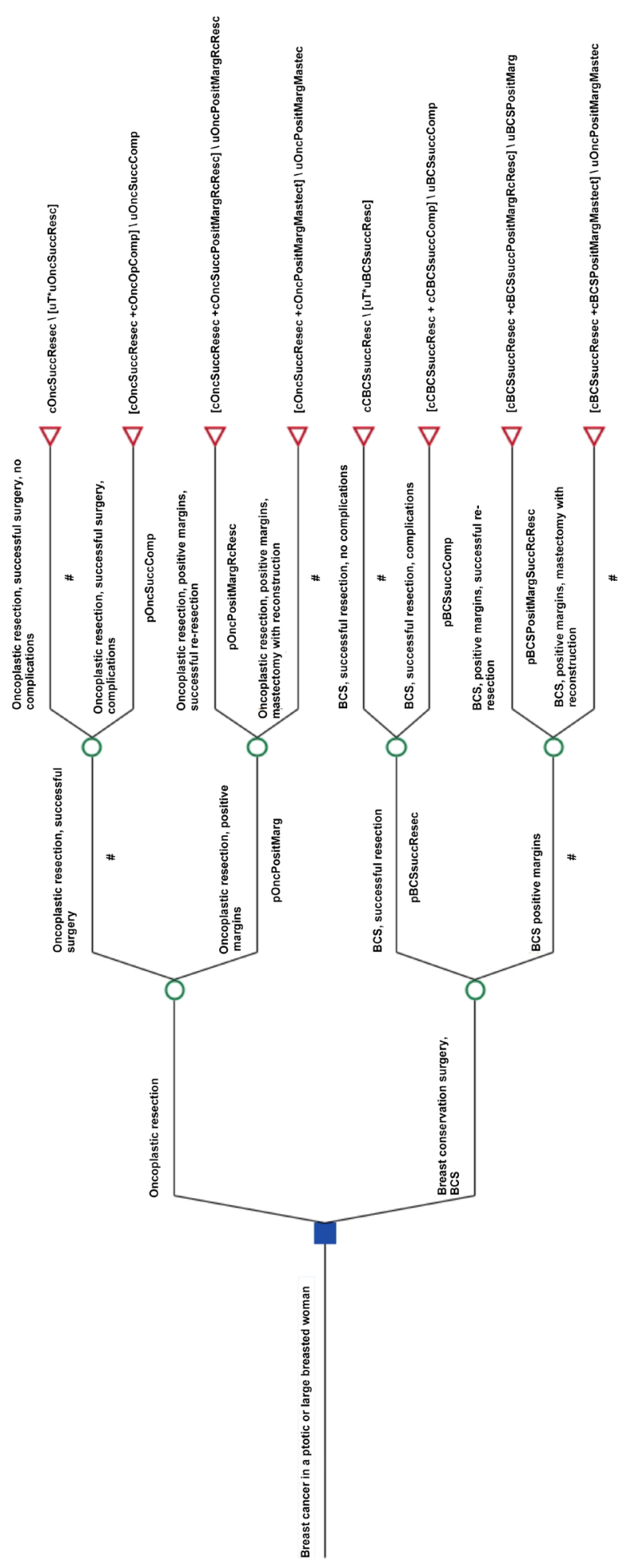

Figure 1. Decision tree comparing conservative surgical options for treating breast cancer in ptotic or large breasted women. Oncoplastic surgery and breast conservation surgery (standard lumpectomy), the two main branches of the decision model, are compared in terms of their clinical outcomes, defined as "health states", each with their respective associated probabilities, costs and utilities. 
probability of a health state by its cost and QALY. These expected values were summed for both arms of the decision tree to derive the overall expected cost and utility (QALY) for the surgical treatment of breast cancer. The incremental cost-utility ratio (ICUR) was then calculated using the formula below.

ICUR=

(Expected cost of standard lumpectomy - Expected cost of large volume displacement oncoplastic surgery) (Expected QALY of standard lumpectomy - Expected QALY of large volume displacement oncoplastic surgery)

This represents the added cost to prolong a patient's life by one year of perfect health [18]. A novel intervention is "cost effective" if the ICUR is greater than 0 and less than the "willingness to pay (WTP)" for an added year of perfect health which we defined as $\$ 50,000$ as described below [19]:

Cost Effective: ICUR $>0$ and $<\mathrm{WTP}, \mathrm{WTP}=\$ 50,000$

If an arm of the tree is more clinically effective and costs less then this arm or breast cancer treatment choice, by definition, dominates the competing arm or breast cancer treatment choice and the ICUR is negative [20]. One-way sensitivity analysis was performed to determine robustness of the baseline decision analysis by varying the clinical utility scores independently for both oncoplastic surgery and SL (and hence their QALYs) and observing how this affected the ICUR. Where applicable, a threshold analysis was performed showing the threshold point for a variable where the cost-effectiveness may favor the alternate surgical approach. All of the above statistical calculations were performed using TreeAge Pro 2012 (Williamstown, MA). Additionally, to analyze which variables impacted our results, we performed a tornado analysis as well as a Monte-Carlo simulation utilizing high impact variables.

This study was done in accordance with the principles outlined in the Declaration of Helsinki.

\section{Results}

Utility scores with subsequent QALYs and associated costs for each health state are shown in Table 1. Costs increased for each treatment option as more surgeries were needed for issues such as positive margins, etc. For example, LVOS with a positive margin requiring mastectomy and reconstruction incorporated cost for the original operation $\$ 6050.97$ in addition to costs for mastectomy with reconstruction to sum up to the value of $\$ 15,484.20$. From a clinical effectiveness standpoint, the decision tree analysis showed a clinical benefit of 1.74 QALYs favoring LVOS (32.78 QALYs) versus SL (QALY 31.04). Further decision tree analysis showed a cost benefit of $\$ 4550$ favoring SL (\$2333.83) versus LVOS (\$6882.83). The ICUR which compared cost of each treatment option over their clinical effectiveness was valued at \$2609.66 /QALY, deeming large volume oncoplastic surgery cost-effective (Table 2 ). With one way sensitivity analysis, we analyzed possible variation in the probability of successful surgery in LVOS and found LVOS was not cost-effective when its probability leading to successful surgery was less than $61.7 \%$ (or the positive margin rate was above $38.3 \%$ ). 
Table 1. Utilities, costs, and quality-adjusted life-years ${ }^{\star}$.

\begin{tabular}{cccc}
\hline Large Volume Oncoplastic Surgery Health States & Utility & Cost (\$) & QALY \\
\hline Successful Surgery & 92.6 & 6050.97 & 33.43 \\
Positive Margin: Successful Re-resection & 70.2 & 7802 & 33.41 \\
Positive Margin: Mastectomy with Reconstruction & 70.0 & $15,484.20$ & 25.27 \\
QALY, quality-adjusted life-years & & & \\
${ }^{*}$ Baseline cost is derived from Medicare Current \\
$\quad$ Procedural Terminology reimbursement
\end{tabular}

Table 2. Comparison of cost and clinical quality and the calculation of ICUR.

\begin{tabular}{cccccc}
\hline & Cost & $\Delta \mathrm{C}$ & $\mathrm{QALY}$ & $\Delta \mathrm{Q}$ & $\mathrm{ICUR}=(\Delta \mathrm{C} / \Delta \mathrm{Q})$ \\
\hline $\begin{array}{c}\text { Large Volume } \\
\text { Oncoplastic Surgery }\end{array}$ & $\$ 6882.86$ & $\$ 4549.03$ & 32.78 & 1.74 & $\$ 2609.11 / \mathrm{QALY}$ \\
$\begin{array}{c}\text { Standard Lumpectomy } \\
\text { (2333.83 }\end{array}$ & & 31.04 & & \\
\hline
\end{tabular}

Furthermore, LVOS became cost-ineffective when in a successful operation cost exceeded $\$ 91,400$. Additionally, we conducted a Tornado diagram that identified the greatest variable of uncertainty as the utility score (and subsequent clinical effectiveness) of SL (Figure 2(a)). Subsequent single variable sensitivity analysis (Figure 2(b)) showed that at a threshold value utility score of 0.9256 for successful SL surgery, SL would be more cost-effective than LVOS. Further supporting LVOS as cost-effective was the Monte Carlo scatter distribution (Figure $3(\mathrm{a})$ ) after micro-simulation assuming a willingness to pay of $\$ 50,000$. After entering reasonable variation for major impact variables (noted in the Tornado diagram), the Monte Carlo micro simulation favored LVOS $87.4 \%$ of the time even with several cost, effectiveness and probability variables changing at the same time for both treatment options based on standard deviation parameters inputted (Figure 3(b)).

\section{Discussion}

Large volume displacement oncoplastic surgery is well established in the literature [1] [2]. Its practice has spread in Europe and the US typically using a two surgeon team (breast surgeon and plastic surgeon) and less frequently a one surgeon approach (single surgeon trained in both breast and plastic surgery). While LVOS constitutes another option in breast conservation, surgeons still debate whether it directly competes against SL or mastectomy with reconstruction 


\section{Tornado Analysis (Net Benefits)}

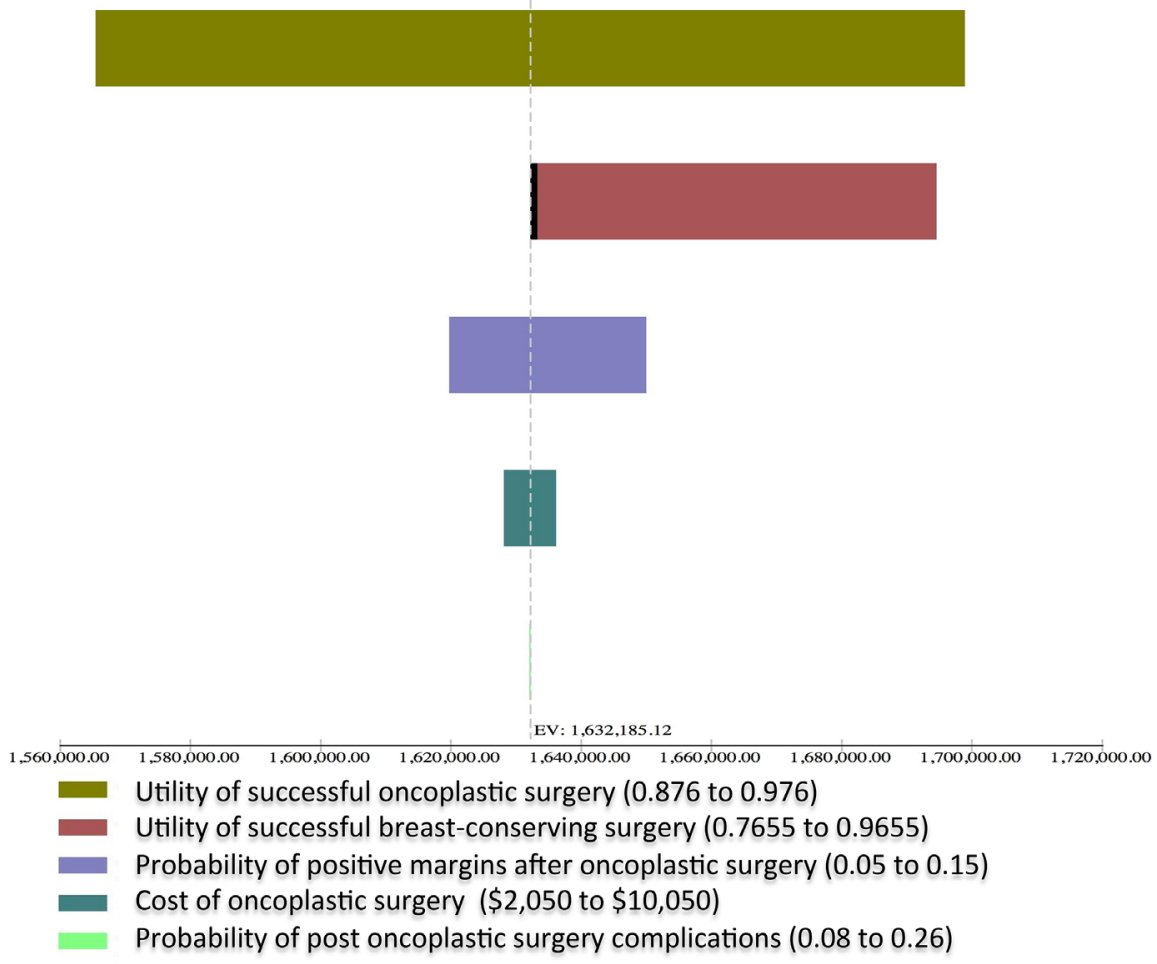

(a)

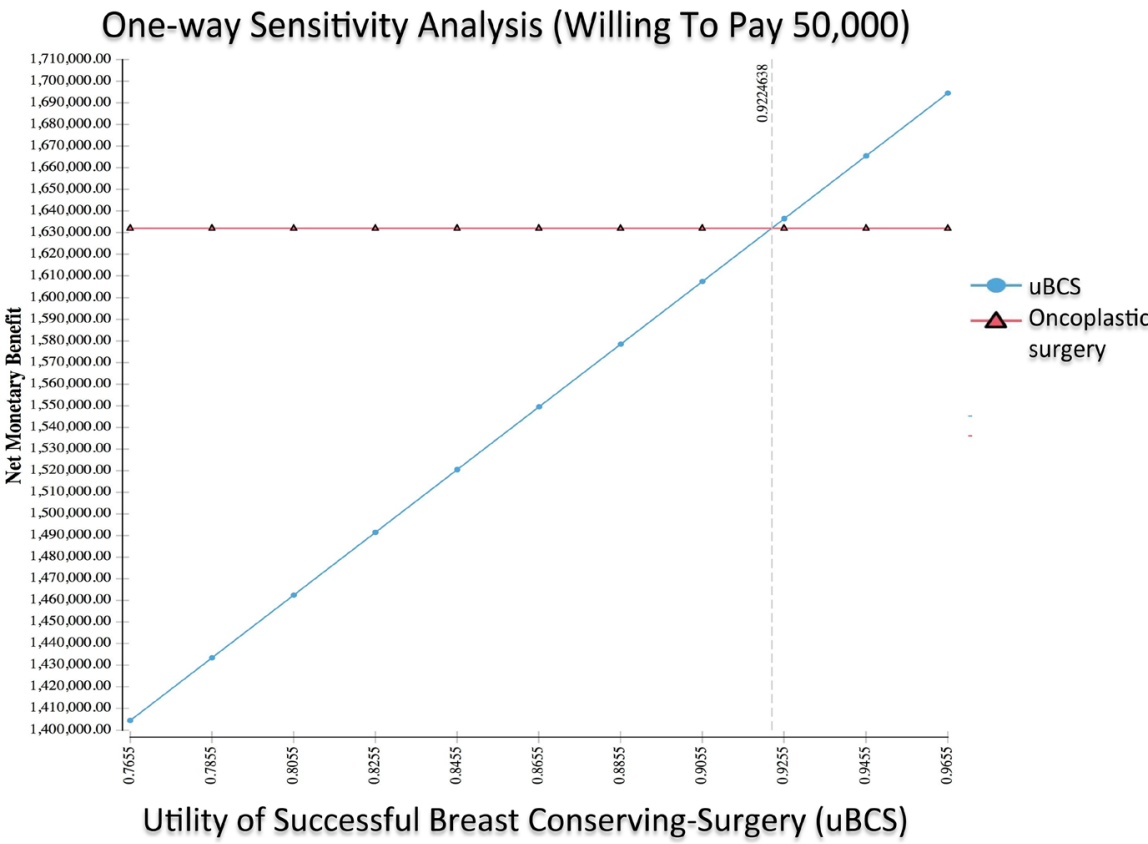

(b)

Figure 2. Tornado diagram and one way sensitivity analysis comparing breast conserving surgical options. (a) Tornado Diagram identifying the greatest variable of uncertainty as the utility score (and subsequent clinical effectiveness) of SL; (b) One way sensitivity analysis demonstrating that at a threshold value utility score of 0.9256 , SL would be more cost-effective than LVOS. 
Incremental Cost-Effectiveness, Oncoplastic resection v. Breast conservation surgery, BCS

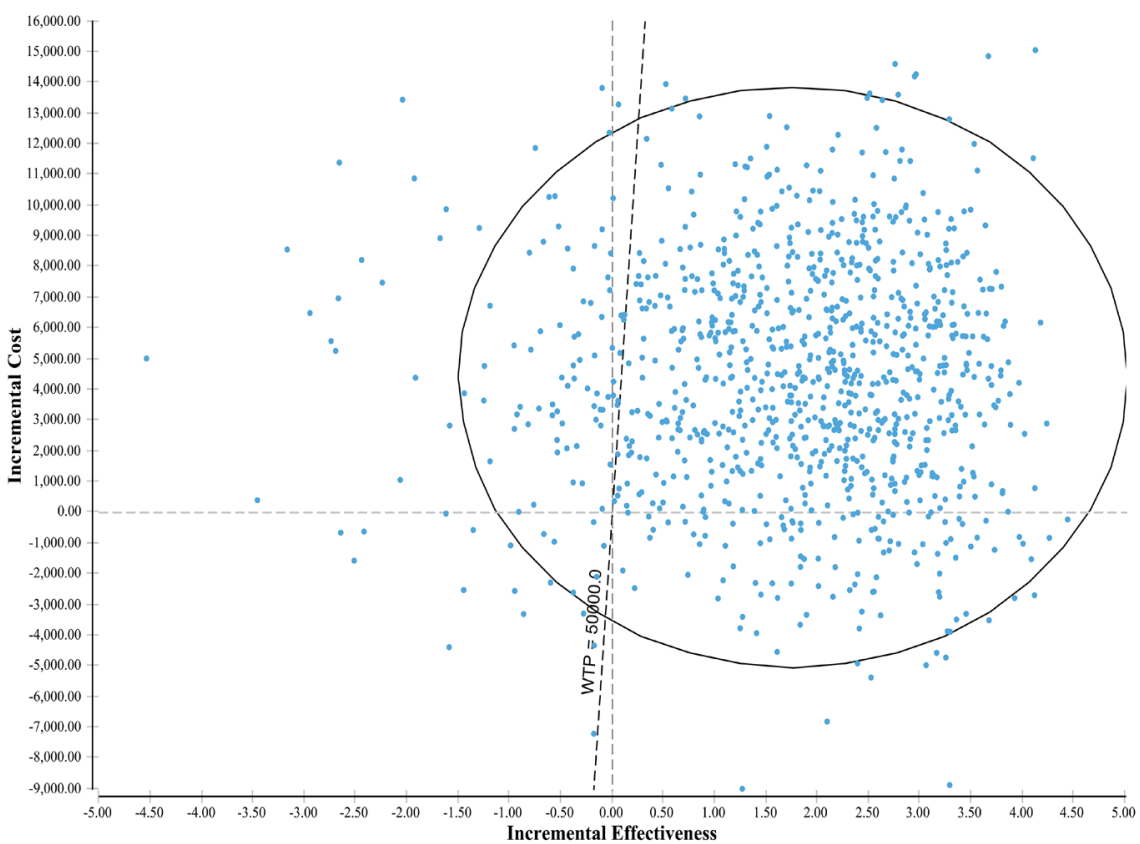

(a)

Monte Carlo Acceptability at WTP (WTP: 50000.0)

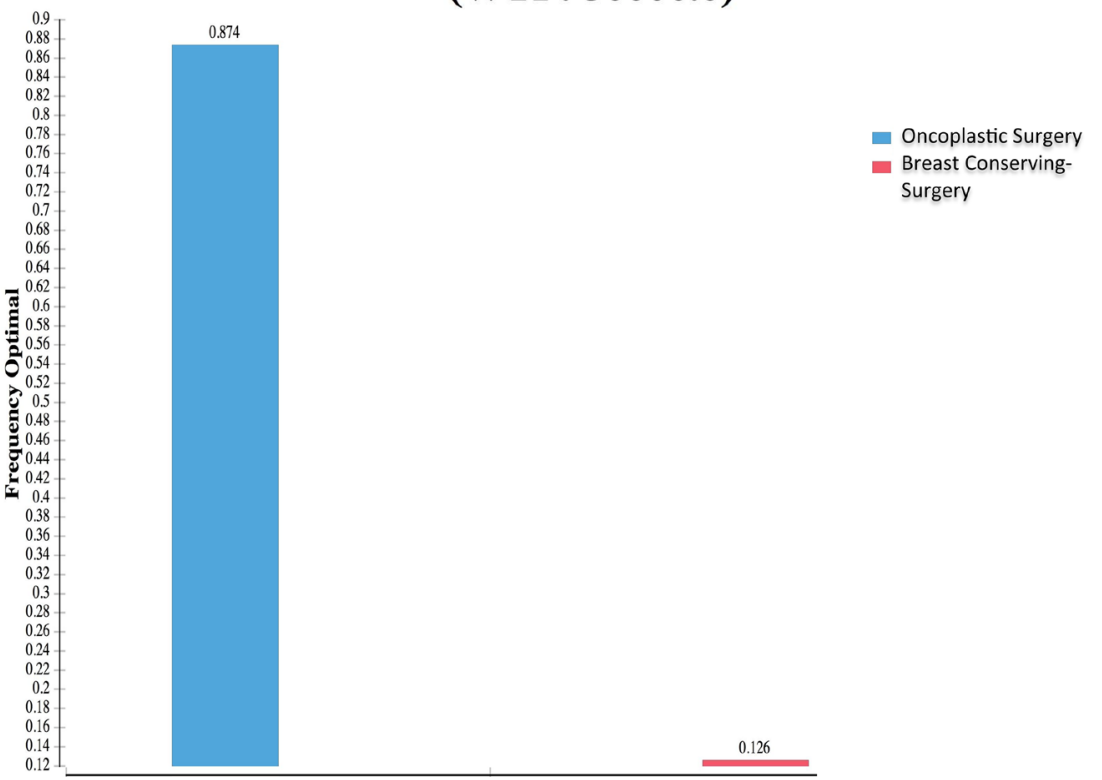

(b)

Figure 3. Monte Carlo analysis. (a) Monte Carlo scatter plot demonstrating LVOS as cost-effective after micro-simulation assuming a willingness to pay of \$50,000; (b) Monte Carlo Acceptability Analysis further supports LVOS as the cost-effective option $87.48 \%$ of the time after entering reasonable variation for major impact variables (noted in the Tornado diagram seen in Figure 2(a).

within the algorithm of breast cancer surgical treatment [21]. From an oncological treatment standpoint, LVOS approach provides a much smaller positive 
margin rate compared to the SL approach [1] [2]. This is further confirmed anecdotally by a similar lower $10 \%$ positive margin rate for LVOS at our institution. Such low positive margin rates in LVOS lead to fewer re-excisions and patients typically report greater satisfaction with fewer operations especially if the cancer is resected successfully after the first operation. Chagpar et al. noted that with greater volumes of tissue removed, the positive margin rate decreases [22]. Large volume displacement oncoplastic surgery allows surgeons to remove large quantities of breast tissue followed by the application of mastopexy or breast reduction techniques to provide aesthetically pleasing, reconstructed breasts. Thus, the significantly smaller positive margin rates for LVOS compared to SL make sense. Standard lumpectomy typically excises less tissue and presumes a seroma cavity which heals through scarring. Standard adjuvant radiation to the lumpectomy site increases scar formation, often leading to poor aesthetic outcomes including nipple retraction and deviation [23]. With LVOS, the lumpectomy cavity is obliterated with tissue rearrangement thus minimizing nipple retraction and skin depression and potentially improving future aesthetics [23]. Such aesthetic advantages of LVOS versus SL likely contribute to the higher utility scores for LVOS versus SL shown in Table 1.

While oncologic postoperative margin and aesthetic advantages favor LVOS over SL, the necessity of operating on both breasts increases the total care costs in the LVOS treatment arm compared to the SL treatment arm. Such treatment options that show either benefits in clinical efficacy with higher costs or poorer clinical outcomes with less cost present a clinical conundrum in which cost-utility analysis proves most useful [18].

Our results depict cost-effectiveness favoring LVOS over SL thus justifying the clinical benefits of LVOS with its lower positive margin rate and improved aesthetics despite the additional costs incurred by operating on both breasts. From an oncologic perspective, the patient benefits as the likelihood of returning to the operating room for re-excision is much lower and adjuvant therapies, including radiation and chemotherapy, can be initiated more expeditiously. The partnership of the breast surgical oncologist and the plastic surgeon facilitates a more definitive tumor ablation by allowing for larger excisions of breast tissue through the utilization of mastopexy or reduction incision pattern techniques to recreate an acceptable final breast form. Presently in North America, the adoption of oncoplastic surgery in certain regions is possibly inhibited by the lack of access to plastic surgeons by breast surgeons interested in oncoplastic surgery [24]. Our results showing the cost-effectiveness of LVOS justify its use as another possible treatment option for the appropriate breast cancer patient.

\section{Limitations}

There are limitations to this study. First, inherent in cost-utility analysis is its reliance on the reliability of the literature in the literature review. Health outcome rates that include positive margin rates and completion mastectomy rates can vary and the literature review gives an overall assessment on rates. We ac- 
knowledge that especially with recent guidelines favoring a $2 \mathrm{~mm}$ margin for DCIS and a no tumor at ink margin for invasive breast cancer [25] [26] past literature reviews and meta-analyses can be inconsistent. Nevertheless, there is consistent meta-analysis data showing LVOS has a lower positive margin rate than SL which makes anatomical sense since LVOS removes larger volumes of tissue. We also compensated for these uncertainties by running the Monte Carlo simulation with varied distributions of several variables (including probabilities for positive margins) that still showed the greater cost-effective option as LVOS. Nevertheless, inherent flaws are present when pooling data from a literature review given the variability of data collection, patients being operated on and surgeons performing the surgeries. A second imitation involves the use of a third party payer perspective. A societal perspective was not performed, and extrapolating conclusions in such would be outside the scope of our analysis. A societal view would have to incorporate the total costs to the patient which would include indirect costs. Nevertheless, the third party payer perspective is a well established and empirically used [9] [10] [11] [12] [27] [28]. Approach in cost-utlity analysis and is particularly applicable to the US health care system. Third, we did not specifically incorporate radiation therapy into our calculations given that both treatment options would require radiation. Thus, there would be no cost advantage in either arm of the decision analysis. However, there may have been a clinical advantage for oncoplastic surgery not appreciated without incorporating radiation therapy since the potential of nipple inversion after radiation to a lumpectomy defect sometimes seen in long term followup is not present in LVOS since there is no dead space after the large volume rearrangement of tissue [29]. Nevertheless, this would only strengthen our conclusion favoring LVOS as cost-effective. Lastly, these results do not advocate for the indiscriminontory use of oncoplastic surgery. For example, an appropriate breast cancer patient who would benefit from LVOS would be one with symptomatically large breasts causing classic symptoms of neck pain, back pain, shoulder pain, and inframammary rash and such surgery would not only remove her cancer but also help with her macromastia symptoms. The goal of this study is to demonstrate that a large volume displacement oncoplastic approach to breast cancer is one that is reasonable and cost-effective in the appropriate patient.

\section{Conclusion}

Large volume displacement oncoplastic surgery is a cost-effective treatment option in breast conservation surgery when treating breast cancer in large breasted women. Its clinical advantages that include a low positive margin rate justify its increased costs and it may be considered as yet another treatment choice in a surgeon's armamentarium.

\section{References}

[1] De La Cruz, L., Blankenship, S.A., et al. (2016) Outcomes after Oncoplastic 
Breast-Conserving Surgery in Breast Cancer Patients: A Systematic Literature Review. Annals of Surgical Oncology, 23, 3247-3258.

https://doi.org/10.1245/s10434-016-5313-1

[2] Losken, A., Dugal, C.S., Styblo, T.M. and Carlson, G.W. (2014) A Meta-Analysis Comparing Breast Conservation Therapy Alone to the Oncoplastic Technique. Annals of Plastic Surgery, 72, 145-149. https://doi.org/10.1097/SAP.0b013e3182605598

[3] Pyfer, B., Chatterjee, A., Chen, L., et al. (2016) Early Postoperative Outcomes in Breast Conservation Surgery versus Simple Mastectomy with Implant Reconstruction: A NSQIP Analysis of 11,645 Patients. Annals of Surgical Oncology, 23, 92-98. https://doi.org/10.1245/s10434-015-4770-2

[4] Kronowitz, S.J., Hunt, K.K., Kuerer, H.M., et al. (2007) Practical Guidelines for Repair of Partial Mastectomy Defects Using the Breast Reduction Technique in Patients Undergoing Breast Conservation Therapy. Plastic and Reconstructive Surgery, 120, 1755-1768. https://doi.org/10.1097/01.prs.0000287130.77835.f6

[5] Losken, A., Styblo, T.M., Carlson, G.W., Jones, G.E. and Amerson, B.J. (2007) Management Algorithm and Outcome Evaluation of Partial Mastectomy Defects Treated Using Reduction or Mastopexy Techniques. Annals of Plastic Surgery, 59, 235-242. https://doi.org/10.1097/SAP.0b013e31802ec6d1

[6] Cavaliere, C.M. and Chung, K.C. (2010) A Cost-Utility Analysis of Nonsurgical Management, Total Wrist Arthroplasty, and Total Wrist Arthrodesis in Rheumatoid Arthritis. Journal of Hand Surgery-American, 35, 379-391. https://doi.org/10.1016/j.jhsa.2009.12.013

[7] Chung, K.C., Saddawi-Konefka, D., Haase, S.C. and Kaul, G. (2009) A Cost-Utility Analysis of Amputation versus Salvage for Gustilo Type IIIB and IIIC Open Tibial Fractures. Plastic and Reconstructive Surgery, 124, 1965-1973. https://doi.org/10.1097/PRS.0b013e3181bcf156

[8] Chatterjee, A., Krishnan, N.M. and Rosen, J.M. (2015) Complex Ventral Hernia Repair using Components Separation with or without Biologic Mesh: A Cost-Utility Analysis. Annals of Plastic Surgery, 74, 471-478. https://doi.org/10.1097/SAP.0b013e31829fd306

[9] Chatterjee, A., Krishnan, N.M. and Rosen, J.M. (2014) Complex Ventral Hernia Repair Using Components Separation with or without Synthetic Mesh: A Cost-Utility Analysis. Plastic and Reconstructive Surgery, 133, 137-146.

https://doi.org/10.1097/01.prs.0000436835.96194.79

[10] Chatterjee, A., Krishnan, N.M., Van Vliet, M.M., Powell, S.G., Rosen, J.M. and Ridgway, E.B. (2013) A Comparison of Free Autologous Breast Reconstruction with and without the Use of Laser-Assisted Indocyanine Green Angiography: A Cost-Effectiveness Analysis. Plastic and Reconstructive Surgery, 131, 693e-701e. https://doi.org/10.1097/PRS.0b013e31828659f4

[11] Krishnan, N.M., Chatterjee, A., Rosenkranz, K.M., Powell, S.G., Nigriny, J.F. and Vidal, D.C. (2014) The Cost Effectiveness of Acellular Dermal Matrix in Expander-Implant Immediate Breast Reconstruction. Journal of Plastic, Reconstructive \& Aesthetic Surgery, 67, 468-476. https://doi.org/10.1016/j.bjps.2013.12.035

[12] Krishnan, N.M., Chatterjee, A., Van Vliet, M.M., Powell, S.G., Rosen, J.M. and Nigriny, J.F. (2013) A Comparison of Acellular Dermal Matrix to Autologous Dermal Flaps in Single-Stage, Implant-Based Immediate Breast Reconstruction: A Cost-Effectiveness Analysis. Plastic and Reconstructive Surgery, 131, 953-961. https://doi.org/10.1097/PRS.0b013e3182865a24

[13] Thoma, A., Khuthaila, D., Rockwell, G. and Veltri, K. (2003) Cost-Utility Analysis 
Comparing Free and Pedicled TRAM Flap for Breast Reconstruction. Microsurgery, 23, 287-295. https://doi.org/10.1002/micr.10138

[14] Ziolkowski, N.I., Voineskos, S.H., Ignacy, T.A. and Thoma, A. (2013) Systematic Review of Economic Evaluations in Plastic Surgery. Plastic and Reconstructive Surgery, 132, 191-203. https://doi.org/10.1097/PRS.0b013e318290f8f8

[15] Ingenix (2012) DRG Expert. ICD-10 Edition, Ingenix.

[16] Services UDoHaH (2012) Physician Fee Schedule Search. Services CfMaM.

[17] Haloua, M.H., Krekel, N.M., Winters, H.A., et al. (2013) A Systematic Review of Oncoplastic Breast-Conserving Surgery: Current Weaknesses and Future Prospects. Annals of Surgery, 257, 609-620. https://doi.org/10.1097/SLA.0b013e3182888782

[18] Thoma, A., Veltri, K., Khuthaila, D., Rockwell, G. and Duku, E. (2004) Comparison of the Deep Inferior Epigastric Perforator Flap and Free Transverse Rectus Abdominis Myocutaneous Flap in Postmastectomy Reconstruction: A Cost-Effectiveness Analysis. Plastic and Reconstructive Surgery, 113, 1650-1661. https://doi.org/10.1097/01.PRS.0000117196.61020.FD

[19] Grosse, S.D. (2008) Assessing Cost-Effectiveness in Healthcare: History of the \$50,000 per QALY Threshold. Expert Review of Pharmacoeconomics \& Outcomes Research, 8, 165-178. https://doi.org/10.1586/14737167.8.2.165

[20] Chen, N.C., Shauver, M.J. and Chung, K.C. (2009) A Primer on Use of Decision Analysis Methodology in Hand Surgery. Journal of Hand Surgery, 34, 983-990. https://doi.org/10.1016/j.jhsa.2009.03.005

[21] Mansell, J., Weiler-Mithoff, E., Martin, J., et al. (2017) How to Compare the Oncological Safety of Oncoplastic Breast Conservation Surgery-To Wide Local Excision or Mastectomy? Breast, 24, 497-501. https://doi.org/10.1016/j.breast.2015.05.003

[22] Chagpar, A.B., Killelea, B.K., Tsangaris, T.N., et al. (2015) A Randomized, Controlled Trial of Cavity Shave Margins in Breast Cancer. The New England Journal of Medicine, 373, 503-510. https://doi.org/10.1056/NEJMoa1504473

[23] Clough, K.B., Ihrai, T., Oden, S., Kaufman, G., Massey, E. and Nos, C. (2012) Oncoplastic Surgery for Breast Cancer Based on Tumour Location and a Quadrant-per-Quadrant Atlas. British Journal of Surgery, 99, 1389-1395. https://doi.org/10.1002/bjs.8877

[24] Maxwell, M.J., Roberts, M.A., et al. (2016) Current Practices and Barriers to the Integration of Oncoplastic Breast Surgery: A Canadian Perspective. Annals of Surgical Oncology, 23, 3259-3265.

[25] Morrow, M., Van Zee, K.J., Solin, L.J., et al. (2016) Society of Surgical Oncology-American Society for Radiation Oncology-American Society of Clinical Oncology Consensus Guideline on Margins for Breast-Conserving Surgery with Whole-Breast Irradiation in Ductal Carcinoma in Situ. Annals of Surgical Oncology, 23, 3801-3810. https://doi.org/10.1245/s10434-016-5449-Z

[26] Moran, M.S., Schnitt, S.J., Giuliano, A.E., et al. (2014) Society of Surgical Oncology-American Society for Radiation Oncology Consensus Guideline on Margins for Breast-Conserving Surgery with Whole-Breast Irradiation in Stages I and II Invasive Breast Cancer. Journal of Clinical Oncology, 32, 1507-1515. https://doi.org/10.1245/s10434-014-3481-4

[27] Chatterjee, A., Krishnan, N.M. and Rosen, J.M. (2014) Complex Ventral Hernia Repair using Components Separation with or without Biologic Mesh: A Cost-Utility Analysis: Reply. Annals of Plastic Surgery, 72, 610-611.

https://doi.org/10.1097/SAP.0000000000000140 
[28] Chatterjee, A., Macarios, D., Griffin, L., et al. (2015) Cost-Utility Analysis: Sartorius Flap versus Negative Pressure Therapy for Infected Vascular Groin Graft Management. Plastic and Reconstructive Surgery, 3, e566. https://doi.org/10.1097/GOX.0000000000000551

[29] Anderson, B.O., Masetti, R. and Silverstein, M.J. (2005) Oncoplastic Approaches to Partial Mastectomy: An Overview of Volume-Displacement Techniques. The Lancet Oncology, 6, 145-157. https://doi.org/10.1016/S1470-2045(05)01765-1 